\title{
Charm quark evolution in the QGP medium
}

\section{YOUNUS, Mohammed*}

Bose Institute, Kolkata, India

Email:younus.presi@gmail.com

\section{SRIVASTAVA, Dinesh}

Variable Energy Cyclotron Centre, Kolkata, India

Email:dinesh@vecc.gov.in

\begin{abstract}
Here we have tried to present results on charm quark energy and momentum evolution using two different approaches. The first approach is the use of phenomenological models of charm collisional energy loss due to elastic scattering with thermalized partons and medium induced radiation of gluons by charm due to inelastic scattering. We have compared our results with latest data from LHC-ALICE experiments at $\sqrt{s}=2.76 \mathrm{TeV} /$ nucleon. In the second approach, we have used transport calculations of parton cascade model-VNI/BMS and have shown path length dependence of charm quark energy loss.
\end{abstract}

7th International Conference on Physics and Astrophysics of Quark Gluon Plasma

1-5 February, 2015

Kolkata, India

* Speaker. 


\section{Introduction}

In relativistic heavy ion collisions, charm quark because of its large mass $(1.5 \mathrm{GeV})$, are mostly produced in early phase when parton momenta are high and the thermalized medium or QGP is not formed yet. Large momentum transfer requirement ensures that small number of charms are produced and $\mathrm{pQCD}$ can be applied in its production mechanisms. This also ensures that its production rate is almost negligible in the thermalized phase [1]. Hence it serves as excellent probe and the information on the properties of quark gluon plasma can be obtained via charm quark transport coefficients which in turn depend upon its rate of interaction with the thermalized medium partons. The following sections would deal with evolution of charm quark energy and momentum via its collision and radiation in QGP medium.

\section{Charm production and energy loss}

The charm production in heavy ion collision can be written as [2],

$$
\frac{d N_{A A}}{d^{2} p_{T} d y}=T_{A A} \frac{d \sigma_{p p}}{d^{2} p_{T} d y}
$$

where,

$$
\begin{aligned}
\frac{d \sigma_{p p}}{d y_{1} d y_{2} d^{2} p_{T}} & =2 x_{1} x_{2} \sum_{i j}\left[f_{i}^{(1)}\left(x_{1}, Q^{2}\right) f_{j}^{(2)}\left(x_{2}, Q^{2}\right) \frac{d \hat{\sigma}_{i j}(\hat{s}, \hat{t}, \hat{u})}{d \hat{t}}\right. \\
& \left.+f_{j}^{(1)}\left(x_{1}, Q^{2}\right) f_{i}^{(2)}\left(x_{2}, Q^{2}\right) \frac{d \hat{\sigma}_{j i}(\hat{s}, \hat{u}, \hat{t})}{d \hat{t}}\right] /\left(1+\delta_{i j}\right)
\end{aligned}
$$

where $\mathrm{i}$ and $\mathrm{j}$ are the interacting partons and $f_{i}$ and $f_{j}$ are the partonic structure functions, and $x_{1}$ and $x_{2}$ are the momentum fractions of the parent nucleons carried by the interacting partons.

\subsection{Collisional energy loss}

Charm quarks traveling through qgp medium, undergo collisional energy loss via elastic scattering with medium partons. Since medium partons are considered to be in local thermal equilibrium, the collisional energy loss per unit length traveled in the medium is found to be a function of charm energy and the medium temperature and can be approximately shown to be [3,4],

$$
\frac{d E_{\text {coll }}}{d x}=\pi \alpha_{s}^{2} T^{2}\left[\left(1+\frac{N_{f}}{6}\right) \cdot \ln \frac{E_{p}(x) T}{\mu_{D}^{2}}+\frac{3}{5} \ln \frac{E_{p}(x) T}{M_{c}^{2}}+c\left(n_{f}\right)\right]
$$

\subsection{Radiative energy loss}

The charm quark upon scattering with the medium may obtain some virtuality and thus lose this virtuality via medium induced radiation. This in turn also makes charm quark lose a part of its energy and momentum. In the region of coherent emission of gluon or LPM effect, the charm radiative energy loss per unit length can be approximately shown to be $[5,6,4]$ :

$$
\frac{d E_{\text {rad }}}{d x}=\frac{C_{A}}{\pi} \alpha_{s}^{3} \rho_{q g p} \sqrt{\hat{q} L^{2}} \cdot \int_{t_{\min }}^{t_{\max }} d \hat{t} \frac{d \sigma}{d \hat{t}} \int_{\omega_{\min }}^{\omega_{\max }} d \omega \frac{1}{\omega^{1 / 2}} \int_{\eta_{\min }}^{\eta_{\max }} d \eta D(\eta)
$$


' $\omega$ ' is the energy of the radiated gluon and ' $D(\eta)^{\prime}$ is the dead cone factor. The limits of the integration for the scattering processes above are calculated to show;

$$
\begin{array}{r}
t_{\text {min }}=\omega_{\text {min }}^{2}=\mu_{D}^{2}=4 \pi \alpha_{s} \cdot T^{2} \\
t_{\text {max }}=\frac{3 E T}{2}-\frac{M^{2}}{2}+\frac{M^{4}}{64 p_{T} T} \ln \left[\frac{M^{2}+6 E T+6 p_{T} T}{M^{2}+6 E T-6 p_{T} T}\right] \\
\omega_{\max }=\left[\int_{t_{\min }}^{t_{\max }} d \hat{t} \cdot \hat{t} \cdot \frac{d \hat{\sigma}}{d \hat{t}}\right]^{1 / 2} \\
\eta_{\min } / \eta_{\max }=(-/+) \ln \left(\frac{\omega_{\max }}{\mu_{D}}+\sqrt{\frac{\omega_{\max }^{2}}{\mu_{D}^{2}}-1}\right)
\end{array}
$$

Then the total energy loss of charm quark is considered to be the sum of the energy loss due to collision and radiation. Cosequently charm ' $R_{A A}$ ' and ' $v_{2}$ ' are calculated for different centrality classes $[4,7,8,9,10]$.

\section{Charm evolution}

In another approach charm energy evolution can be studied using transport equation due to parton cascade model or VNI/BMS. The momentum and energy of charm propagating through a block/brick of finite sized qgp like medium can be traced for various path lengths and fixed medium temperatures. We insert a charm quark with the four momentum $p^{\mu}=\left\{0,0, p_{z}, E=\sqrt{p_{z}^{2}+M_{c}^{2}}\right\}$, into the box and let it evolve according to the Relativistic Boltzmann Equation given by,

$$
p^{\mu} \frac{\partial F_{k}(x, \vec{p})}{\partial x^{\mu}}=\sum_{\text {processes:i }} C_{i}[F]
$$

where $F_{k}(x, \vec{p})$ is the single particle phase space distribution and the collision term on r.h.s. is a non-linear functional of phase space distribution terms inside an integral [11].

We have included the matrix elements for all $2 \rightarrow 2$ binary elastic scattering processes for charm interaction with gluons or light quarks $(u, d, s)$ and $2 \rightarrow 3$ process for radiative (brehmsstrahlung) corrections after each scattering and finally we have calculated charm energy as the function of distance traveled in the medium for a given temperature and for various initial charm energies. This particular mode gives us an opportunity to study charm quark energy and momentum evolution in infinite QGP like medium. A more realistic approach would be to include expanding medium with temperature variations.

\section{Results and discussions}

The Fig. 1 shows plots for $R_{A A}$ and $v_{2}$ for two different centralities. The model results agree with the data [12] to certain extent and are well within error bars. We have shown our results for two different centrality classes namely $0-10 \%$ and $30-50 \%$ centralities. However the $v_{2}$ results show considerable differences from data [12]. We feel that it may be due to the absence of any transverse expansion in our model. The expanding medium will be taken care of using hydrodynamical simulatons for the quark gluon plasma in our future publcations. In the Fig. 2 we showed path length 

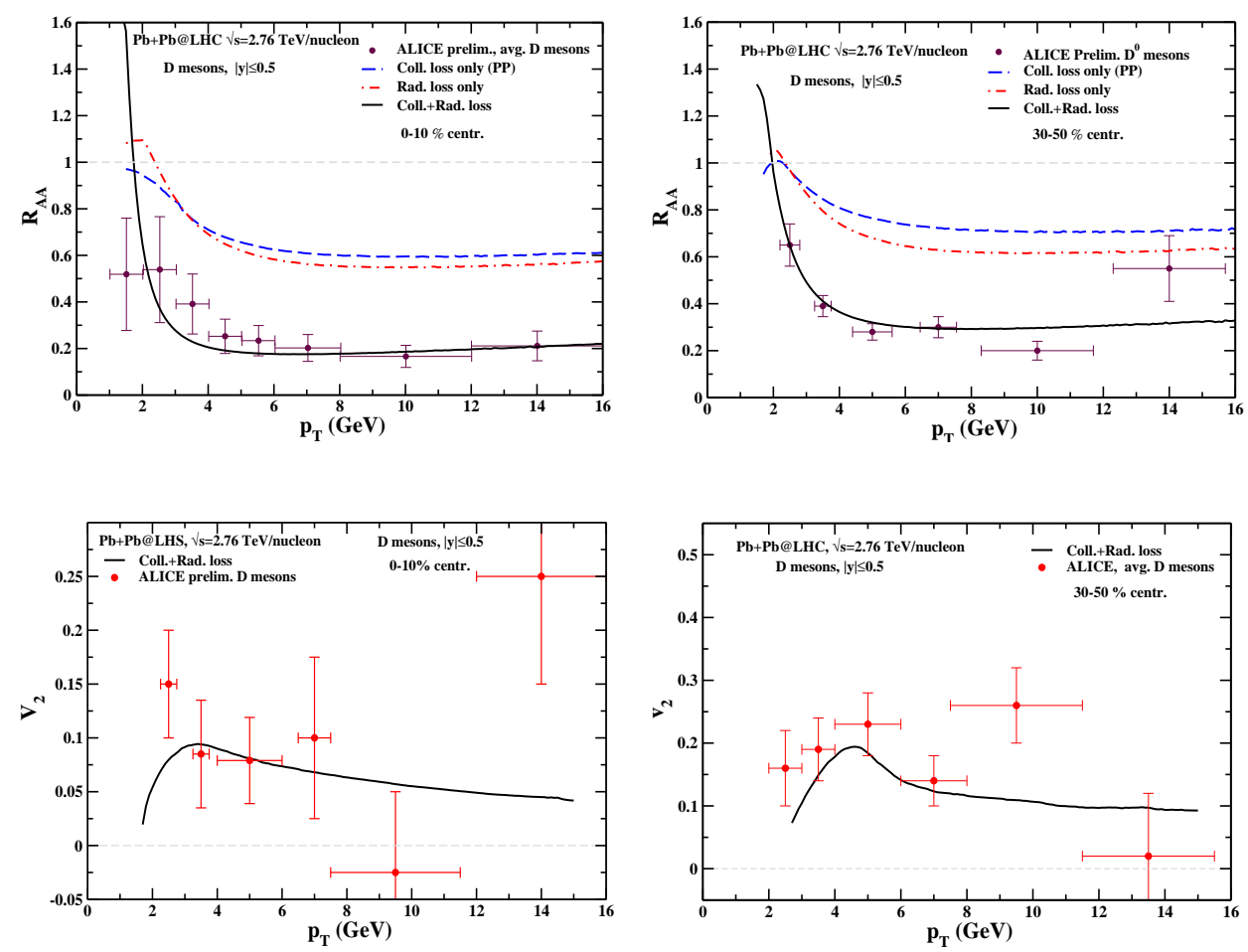

Figure 1: Nuclear modification factor, ' $R_{A A}$ ' and ' $v_{2}$ ', for $0-10 \%$ centr. and $30-50 \%$ centr.

dependence of energy of a charm by tracking it over a finite path length within the QGP brick using transport calculations of parton cascade model of VNI/BMS $[13,11]$. The calculation is done at constant temperature of $\mathrm{T}=350 \mathrm{MeV}$ which is realizable at RHIC like systems and two different charm energies of $16 \mathrm{GeV}$ and $50 \mathrm{GeV}$ have been used. As the next step more realistic expanding medium scenario will be incorporated. The Fig. 2 contain plots for collisional and radiative energy loss of charm separately and are compared with the analytical calculations due to [7]
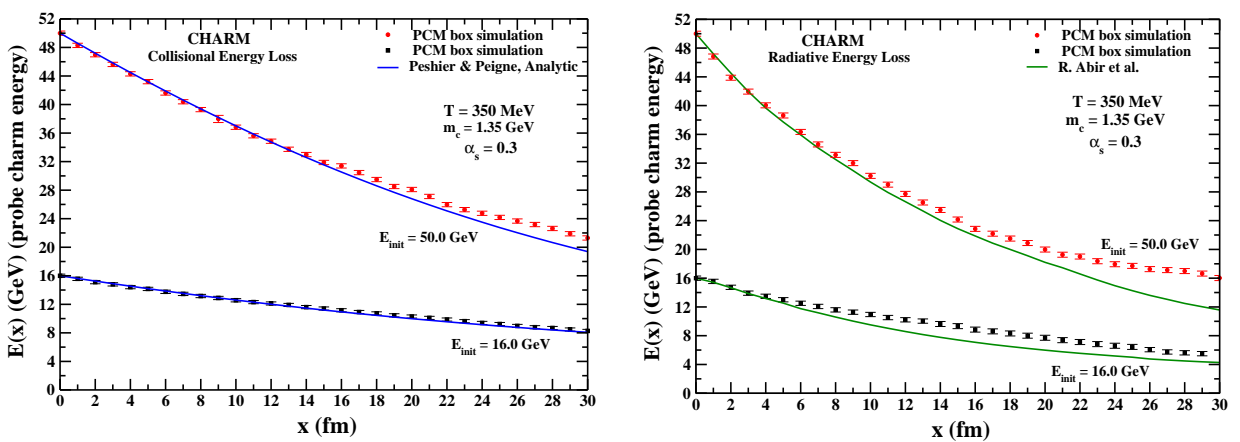

Figure 2: charm energy evolution with path length traveled in QGP 


\section{Conclusions}

The present work gives a comparative study of charm energy loss between a few phenomenological models and transport calculation of parton cascade model/VNI-BMS. As the result charm energy loss per unit length shown and D mesons $R_{A A}$ and $v_{2}$ are calculated are shown for $\sqrt{s}=2.76$ $\mathrm{TeV} /$ nucleon.

\section{Acknowledgement}

One of us(MY) would like to thank the organizers of ICPA-QGP for providing the opportunity of participating and writing this manuscript.

\section{References}

[1] Z. Lin and M. Gyulassy, Open charm as a probe of preequilibrium dynamics in nuclear collisions, Phys. Rev. C 51, no.4, (1995) 2177 [nucl-th / 9409007 ]; M. Younus and D. K. Srivastava, Heavy quark production in relativistic heavy ion collisions, J. Phys. G: Nucl. Part. Phys. 37, (2010) 115006 [arXiv: 1008.1120 [nucl-th]].

[2] U. Jamil and D. K. Srivastava, Nuclear suppression of heavy quark production at forward rapidities in relativistic heavy ion collisions, J. Phys. G: Nucl. Part. Phys. 37, (2010) 085106 [arXiv:1005.1208[nucl-th]].

[3] S. Peigne and A. Peshier, Collisional energy loss of a fast heavy quark in a quark gluon plasma, Phys. Rev. D 77, (2008) 114017 [arXiv: 0806.0242 [hep-ph]].

[4] M. Younus, Nuclear modification and azimuthal anisotropy of D mesons produced in relativistic heavy ion collision at $\sqrt{s}=2.76 \mathrm{~A} \mathrm{TeV}$, (2015) [arXiv: 1503.06936 [nucl-th] ].

[5] R. Baier et al., Radiative energy loss of high-energy quarks and gluons in a finite volume quark-gluon plasma, Nucl. Phys. B 483, (1997) 291 [hep-ph/9607355].

[6] L. D. Landau and I. P. Pomeranchuk, Limits of applicability of the theory of bremsstrahlung electrons and pair production at high energies, Dokl. Akad. Nauk. Ser. Fiz., 92, (1954) 535; A. B. Migdal, Bremsstrahlung and pair production in condensed media at high-energies, Phys. Rev. 103, (1956) 1811; Y. L. Dokshitzer and D. E. Kharzeev, Heavy quark colorimetry of QCD matter, Phys. Lett. B 519, (2001) 199 [hep-ph/0106202].

[7] R. Abir et al., Heavy quark energy loss and D-Mesons at RHIC and LHC energies, Phys. Lett. B 715, (2012) 183 [arXiv: 1203.5221 [hep-ph] ]; M. Younus et al., Empirical determination of charm quark energy loss and its consequences for azimuthal anisotropy, J. Phys. G: Nucl. Part. Phys. 39, (2012) 095003 [arXiv:1204.5356 [nucl-th]].

[8] S. Das et al., Heavy-flavour in-medium momentum evolution: Langevin versus Boltzmann approach, Phys. Rev. C 90, (2014) 044901 [arXiv: 1312.6857 [nucl-th]].

[9] S. Cao et al., Heavy quark dynamics and hadronization i ultrarelativistic heavy-ion collisions: Collisional versus radiative energy loss, Phys. Rev. C 88, (2013) 044907 [arXiv:1308.0617 [nucl-th] ]; M. He et al., Non-perturbative heavy flavor transport at RHIC and LHC Nucl. Phys. A 910, (2013) 409 [arXiv: 1208.0256 [nucl-th] ]. 
[10] M. Nahrgang et al., Heavy quark quenching from RHIC to LHC and the consequences of gluon damping, Nucl. Phys. A 904, (2013) 992c [arXiv: 1211.2281 [hep-ph] ]; J. Uphoff et al., Elliptic flow and nuclear modification factor within a partonic transport model, Nucl. Phys. A 931, (2014) 937.

[11] M. Younus et al., Charm quark energy loss in infinite QCD matter using a parton cascade model, Phys. Rev. C 91,(2015) 024912 [arXiv:1309.1276 [nucl-th]].

[12] B. B. Abelev et al. [Alice Collaboration], Azimuthal anisotropy of $\mathrm{D}$ meson production in $\mathrm{Pb}-\mathrm{Pb}$ collisions at $\sqrt{s_{N N}}=2.76 \mathrm{TeV}$, Phys. Rev. C 90, no. 3, (2014) 034904 [arXiv: 1405.2001 [nucl-ex] ]; A. Festanti [ALICE Collaboration], Measurement of D meson azimuthal anisotropy in Pb-Pb collisions at $\sqrt{s_{N N}}=2.76 \mathrm{TeV}$ with ALICE, Nuovo Cim. C 037, no. 01, (2014) 287.

[13] S. A. Bass et al., RHIC physics with parton cascade model, J. Phys. G: Nucl. Part. Phys. 30, (2004) S1283 [nucl-th/ 0403033 ]. 